Proceedings of the 41st Polish Seminar on Positron Annihilation, Lublin, September 9-13, 2013

\title{
Hydrogen-Induced Defects in Palladium
}

\author{
O. Melikhova*, J. ČížEK AND I. ProchÁzKA \\ Faculty of Mathematics and Physics, Charles University in Prague \\ V Holešovičkách 2, Praha 8, CZ-180 00, Czech Republic
}

\begin{abstract}
In the present work positron annihilation spectroscopy was employed for investigation of hydrogen-induced defects in Pd. Well annealed Pd samples were electrochemically charged with hydrogen and development of defects with increasing hydrogen concentration $x_{\mathrm{H}}$ was investigated. At low concentrations $\left(\alpha\right.$-phase, $\left.x_{\mathrm{H}}<0.017 \mathrm{H} / \mathrm{Pd}\right)$ hydrogen loading introduced vacancies, since absorbed hydrogen segregating at vacancies lowers remarkably the vacancy formation energy. When hydrogen concentration exceeds $0.017 \mathrm{H} / \mathrm{Pd}$, particles of palladium hydride $(\mathrm{PdH})$ are formed. Stress induced by growing PdH particles leads to plastic deformation which generates dislocations and vacancies in the sample.
\end{abstract}

DOI: $10.12693 /$ APhysPolA.125.752

PACS: 78.70.Bj, 61.72.-y

\section{Introduction}

Palladium is widely used as a model system for investigations of hydrogen absorbed in metal lattice. Pd is able to absorb relatively large amount of hydrogen and can be easily charged with hydrogen [1]. There are many reports that hydrogen can be trapped at open volume defects like vacancies [2], dislocations [3], and grain boundaries [4]. This can be explained by positive binding energy of hydrogen to defect, i.e. hydrogen attached to defect exhibits lower energy than hydrogen located at octahedral interstitial site [5]. However, it was found that hydrogen may also create new defects. Fukai and Ōkuma [6] discovered extraordinary high vacancy concentration in $\mathrm{Pd}$ annealed at high hydrogen pressure of 5 GPa. Kirchheim [7] showed that formation of hydrogen-induced defects can be explained by a decrease of defect formation energy by segregating hydrogen. A new term "defactants $=$ DEFect ACTing AgeNTS" [8] was introduced for solutes segregating at defects and lowering defect formation energies in analogy to surfactants which reduce surface energies in liquids. Because of its small size, high mobility and attractive interaction with open volume defects hydrogen seems to be a typical example of defactant and may cause a significant enhancement of the concentration of open volume defects.

In the present work positron annihilation spectroscopy (PAS) was employed for characterization of defects created in Pd electrochemically charged with hydrogen at ambient temperature. PAS is a well established non-destructive method with very high sensitivity to vacancies and other open-volume defects [9] and proved itself to be a very powerful tool for investigations of hydrogen interaction with defects in metals on the atomic scale [10].

\section{Experimental \\ 2.1. Specimens}

Bulk Pd $(99.9 \%)$ specimens $\left(10 \times 10 \mathrm{~mm}^{2}\right.$, thickness $\approx 0.5 \mathrm{~mm}$ ) were firstly annealed $\left(1000^{\circ} \mathrm{C} / 2 \mathrm{~h}\right)$ in vacuum

*corresponding author; e-mail: oksivmel@yahoo.com $\left(10^{-3}\right.$ mbar $)$ to remove virtually all defects introduced during casting and shaping. It is important to have virtually defects-free virgin samples since hydrogen interaction with open volume defects existing in the samples would complicate interpretation of results. The specimens were electrochemically charged with hydrogen in a cell filled with a mixture of $\mathrm{H}_{3} \mathrm{PO}_{4}$ and glycerine (volume ratio 1:2) bubbled with Ar for $24 \mathrm{~h}$ to remove dissolved oxygen. Hydrogen loading was performed at ambient temperature using galvanostat and applying constant current pulses between Pt counter electrode (anode) and $\mathrm{Pd}$ sample (cathode). The current density on the sample was kept at $0.3 \mathrm{~mA} / \mathrm{cm}^{2}$. The hydrogen concentration introduced into the sample was calculated from the transported charge using the Faraday law. The electromotoric force (EMF) [3], i.e. the electrochemical potential between the loaded sample and the reference $\mathrm{Ag} / \mathrm{AgCl}$ electrode, was measured to monitor hydrogen absorption in the sample. Hydrogen concentration estimated from the EMF measurement agrees well with that calculated from the Faraday law.

\subsection{Positron annihilation spectroscopy}

A digital positron lifetime (LT) spectrometer [11] with excellent time resolution of 145 ps (FWHM ${ }^{22} \mathrm{Na}$ ) was employed for PAS investigations. At least $10^{7}$ positron annihilation events were accumulated at each LT spectrum which was subsequently decomposed into individual components using a maximum likelihood procedure [12].

$\mathrm{A}^{22} \mathrm{Na}_{2} \mathrm{CO}_{3}$ positron source $(\approx 1.5 \mathrm{MBq})$ deposited on a $2 \mu \mathrm{m}$ thick Mylar foil was always forming a sandwich with two identically treated Pd specimens. The source contribution in LT spectra consisted of two weak components with lifetimes $\approx 368 \mathrm{ps}$ and $\approx 1.5 \mathrm{~ns}$, and corresponding intensities of $\approx 8 \%$ and $\approx 1 \%$.

\section{Results and discussion}

\subsection{Reference specimens}

Two well defined reference Pd samples were investigated in order to determine the $\mathrm{Pd}$ bulk positron lifetime and the lifetime of positrons trapped at dislocations in $\mathrm{Pd}$. The annealed $\left(1000^{\circ} \mathrm{C} / 2 \mathrm{~h}\right) \mathrm{Pd}$ sample exhibits a single component LT spectrum with the lifetime $\tau_{\mathrm{B}}=(110.1 \pm 0.5)$ ps. Theoretical calculations 
of the bulk positron lifetime for $\mathrm{Pd}$ were performed in Ref. [13] using (i) atomic superposition (ATSUP), i.e. a simple method which uses non-self consistent atomic electron densities, and (ii) linear muffin-tin orbital method within the atomic-spheres approximation (LMTO-ASA), i.e. a method that uses self-consistent electronic densities. Electron-positron correlation was treated either within the local density approximation (LDA) using the parameterization by Boroński and Nieminen [14] or within the generalized gradient approximation (GGA) scheme developed by Barbiellini et al. [15]. It is known that LDA approach overestimates positron trapping rates especially with $d$ electrons leading to positron lifetimes which are too short in particular when the self-consistent electron density is used [15]. The GGA approach is more precise, but it is also more sensitive to details of electron density. Accurate lifetimes are obtained using GGA with self-consistent electron density only. Hence, the LMTO-ASA scheme with the GGA approach for the electronpositron correlation can be considered as the most accurate scheme. Using this approach Campillo Robles et al.[13] obtained the Pd bulk positron lifetime of $113 \mathrm{ps}$ which is in good agreement with the lifetime measured in the annealed specimen. Hence, one can conclude that the annealed sample exhibits very low density of defects and virtually all positrons are annihilated in the free state.

Another Pd sample was cold rolled to the thickness reduction of $50 \%$. The cold rolled sample exhibits a single component spectrum with lifetime $\tau_{\mathrm{D}}=(160.9 \pm 0.3) \mathrm{ps}$ which is significantly longer than the $\mathrm{Pd}$ bulk lifetime but shorter than the lifetime $\tau_{\mathrm{V}}=209 \mathrm{ps}$ calculated for a Pd monovacancy using the LMTO-ASA scheme with the GGA approach [13]. This is typical for positrons trapped at dislocations. Hence one can conclude that in the cold rolled sample virtually all positrons are trapped at dislocations created by plastic deformation (saturated trapping) and $\tau_{\mathrm{D}}$ can be considered as a characteristic lifetime for dislocations in $\mathrm{Pd}$.

\subsection{Hydrogen loaded specimens}

The LT spectra of Pd samples loaded by hydrogen can be well fitted by two exponential components. The dependence of positron lifetimes on the hydrogen concentration $x_{\mathrm{H}}$ introduced into the sample is plotted in Fig. 1. The shorter component with lifetime $\tau_{1}<\tau_{\mathrm{B}}$ represents a contribution of free positrons while the longer component with lifetime $\tau_{2}$ can be attributed to positrons trapped at defects created by hydrogen loading.

The relative intensity $I_{2}$ of the latter component is plotted in Fig. 2. One can see in the figure that hydrogen-induced defects were created already after the first loading step $\left(x_{\mathrm{H}}=0.005\right)$ and their concentration rapidly grows with increasing hydrogen concentration in the sample.

Vertical dashed line in Figs. 1, 2 indicates the maximum hydrogen solubility $x_{\mathrm{H}}=0.017$ in the $\alpha$-phase in $\mathrm{Pd}$ at ambient temperature [1]. Below this concentration hydrogen gradually fills octahedral interstitial sites in fcc $\mathrm{Pd}$ lattice and $\mathrm{Pd}-\mathrm{H}$ system remains a single phase

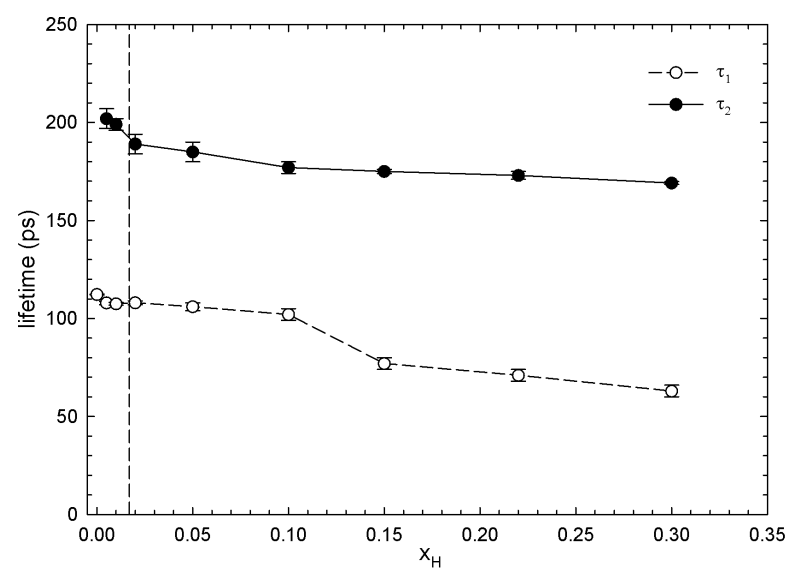

Fig. 1. Two-component decomposition of LT spectra: dependence of positron lifetimes on the hydrogen concentration introduced into the sample.

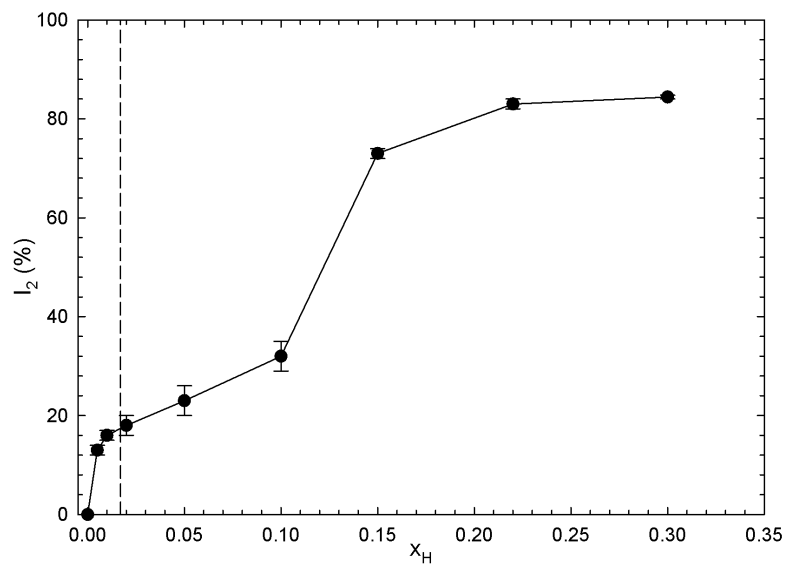

Fig. 2. Two-component decomposition of LT spectra: the intensity $I_{2}$ of positrons trapped at defects plotted as a function of hydrogen concentration.

solid solution (so called $\alpha$-phase). When hydrogen concentration exceeds 0.017 (so called two phase field) palladium hydride $(\mathrm{PdH})$ particles are formed in the sample and $\mathrm{Pd}-\mathrm{H}$ system becomes a mechanical mixture of the $\alpha$-phase and hydrogen-rich $\mathrm{PdH}$. From inspection of Figs. 1, 2 it becomes clear that in the $\alpha$-phase region $\left(x_{\mathrm{H}}<0.017\right) I_{2}$ steeply increases and the lifetime $\tau_{2}$ is approximately $200 \mathrm{ps}$ which is close to the lifetime calculated for $\mathrm{Pd}$ vacancy [13]. Hence, in the $\alpha$-phase region hydrogen loading creates vacancies. Slightly lower value of $\tau_{2}$ with respect to the lifetime $\tau_{\mathrm{V}}=209 \mathrm{ps}$ calculated for Pd monovacancy is likely caused by hydrogen atoms bound to the vacancy [16]. In the two-phase field $\left(x_{\mathrm{H}}>0.017\right)$ the intensity $I_{2}$ increases further and finally tends to saturation at high hydrogen concentrations $x_{\mathrm{H}} \approx 0.30$. This is accompanied by a gradual decrease of the lifetime $\tau_{2}$ down to $\approx 170$ ps. Growing hydride particles emit dislocation loops [17] and stresses induced by the hydride particles, having larger volume than the $\alpha$-phase matrix, cause plastic deformation. Hence at 
$x_{\mathrm{H}}>0.017$ hydrogen loading introduces not only vacancies but also dislocations and positrons trapped at both these two kinds of defects contribute to the component with lifetime $\tau_{2}$. The decrease of $\tau_{2}$ with increasing hydrogen concentration (see Fig. 1) is therefore caused by increasing fraction of positrons trapped at dislocations.

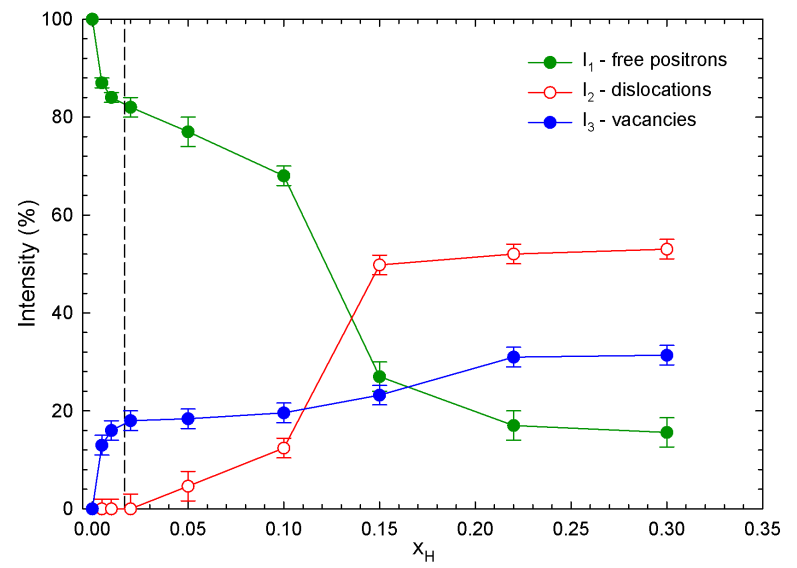

Fig. 3. Three-component decomposition of LT spectra: dependence of relative intensities of LT components on the hydrogen concentration. To reduce the number of fitting parameters the lifetimes of defect components were fixed $\tau_{2}=160 \mathrm{ps}$ (positrons trapped at dislocations) and $\tau_{3}=200 \mathrm{ps}$ (positrons trapped at vacancies).

In order to separate the contributions of positrons trapped at vacancies and dislocations the LT spectra were decomposed into three components. To reduce the number of fitting parameters the lifetime $\tau_{2}$ was fixed at $160 \mathrm{ps}$ and $\tau_{3}$ was fixed at $200 \mathrm{ps}$, i.e. the known lifetimes for positrons trapped at dislocations and vacancies, respectively. The goodness-of-fit characterized by $\chi^{2}$ per degree of freedom was better for the three-component fit with fixed positron lifetimes than for the two component fit. This indicates that the three-component model with vacancies and dislocations provides better descriptions of the experimental data. The relative intensities $I_{1}, I_{2}$, and $I_{3}$ of the components obtained from the three-component decomposition of LT spectra are plotted in Fig. 3 as a function of the hydrogen concentration. One can see in the figure that the intensity $I_{3}$ of positrons trapped at vacancies rises sharply already in the $\alpha$-phase field. In the two-phase field $\left(x_{\mathrm{H}}>0.017\right)$ the increase of $I_{3}$ continues but becomes less pronounced. The dislocation component appears in LT spectrum at $x_{\mathrm{H}}>0.017$ indicating that dislocations are created when formation of $\mathrm{PdH}$ particles takes place.

The concentration of hydrogen-induced vacancies $c_{\mathrm{V}}$ can be calculated from the LT results using the three-state simple trapping model [18]:

$$
c_{\mathrm{V}}=\frac{1}{\nu_{\mathrm{V}}}\left[\frac{I_{2} I_{3}}{I_{1}}\left(\frac{1}{\tau_{3}}-\frac{1}{\tau_{2}}\right)+\frac{I_{3}}{I_{1}}\left(\frac{1}{\tau_{\mathrm{B}}}-\frac{1}{\tau_{3}}\right)\right],
$$

where $\nu_{\mathrm{V}}$ is the specific positron trapping rate to vacancies. The concentration of vacancies calculated from
Eq. (1) using the value $\nu_{\mathrm{V}}=10^{14} \mathrm{~s}^{-1}$, which is typical for vacancies in metals [19], is plotted in Fig. 4.

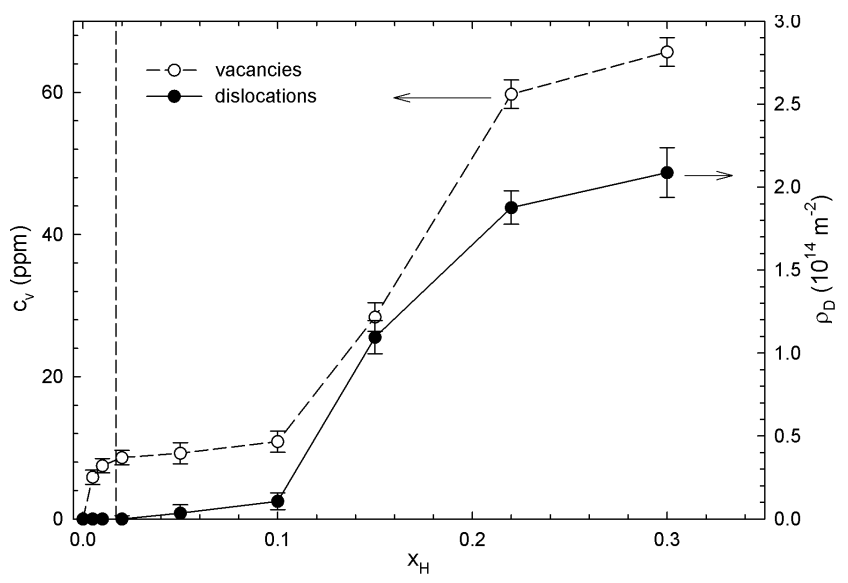

Fig. 4. The concentration of hydrogen-induced vacancies (open points) and dislocation density (full points) plotted as a function of hydrogen concentration in the sample.

The density of dislocations $\rho_{\mathrm{D}}$ can be also calculated from the three-state trapping model

$$
\rho_{\mathrm{D}}=\frac{1}{\nu_{\mathrm{D}}}\left[\frac{I_{2} I_{3}}{I_{1}}\left(\frac{1}{\tau_{2}}-\frac{1}{\tau_{3}}\right)+\frac{I_{2}}{I_{1}}\left(\frac{1}{\tau_{\mathrm{B}}}-\frac{1}{\tau_{2}}\right)\right],
$$

where $\nu_{\mathrm{D}}=0.5 \times 10^{-4} \mathrm{~m}^{2} \mathrm{~s}^{-1}[19]$ is the specific positron trapping rate to dislocations. The dislocation density calculated using Eq. (2) is plotted in Fig. 4 as a function of hydrogen concentration.

From inspection of Fig. 4 one can conclude that in the $\alpha$-phase field $\left(x_{\mathrm{H}}<0.017\right)$ hydrogen loading introduces vacancies since vacancy formation energy is lowered by segregated hydrogen. The equilibrium concentration of vacancies at certain temperature $T$ is given by the expression

$$
c_{\mathrm{V}, \mathrm{eq}}=\mathrm{e}^{S_{\mathrm{f}} / k} \mathrm{e}^{-E_{\mathrm{f}} / k T},
$$

where $S_{\mathrm{f}}$ and $E_{\mathrm{f}}$ denote the vacancy formation entropy and enthalpy, respectively, and $k$ is the Boltzmann constant. Using $S_{\mathrm{f}}=(2-3) k[20]$ and $E_{\mathrm{f}}=1.68 \mathrm{eV}[21]$ one can calculate that the equilibrium vacancy concentration at the ambient temperature in pure $\mathrm{Pd}$ is extremely small and falls in the range $(1-3) \times 10^{-28}$. In the sample loaded by hydrogen within the $\alpha$-phase field the concentration of vacancies increased up to $\approx 8 \times 10^{-7}$, see Fig. 4. Hence, absorbed hydrogen remarkably lowers vacancy formation energy and enhances the concentration of vacancies by more than 20 orders of magnitude. From Eq. (3) one can calculate that the concentration of vacancies $c_{\mathrm{V}} \approx 8 \times 10^{-7}$ measured in the experiment is obtained if the vacancy formation energy is lowered from 1.68 down to $E_{\mathrm{f}} \approx 0.4 \mathrm{eV}$. Thus, hydrogen absorbed in $\mathrm{Pd}$ indeed acts as defactant and decreases the vacancy formation energy roughly by $1.3 \mathrm{eV}$. This value is comparable with lowering of the vacancy formation energy by $1.06 \mathrm{eV}$ determined by Fukai and Ōkuma in a high pressure hydrogen gas loading experiment [6]. 
In the two-phase field $\left(x_{\mathrm{H}}>0.017\right)$ the stresses introduced by hydride particles cause plastic deformation and create dislocations in the sample which is reflected by a rise of the dislocation component in Fig. 3. The dislocation density strongly increases in the range of hydrogen concentrations $x_{\mathrm{H}}=0.1-0.2$ and finally saturates at the value $\rho_{\mathrm{D}} \approx 2 \times 10^{14} \mathrm{~m}^{-2}$ which is rather high dislocation density usually found in heavily deformed metals. Plastic deformation produces not only dislocations but also vacancies which are created by crossing dislocations. In Fig. 4 one can see that the increase of vacancy concentration in the two-phase field is indeed correlated with the increase of dislocation density.

\section{Conclusions}

Defect studies of $\mathrm{Pd}$ specimens electrochemically charged with hydrogen were performed by LT spectroscopy. It was found that vacancy formation energy is significantly lowered by segregating hydrogen. As a consequence hydrogen loading in the $\alpha$-phase region $\left(x_{\mathrm{H}}<0.017\right)$ leads to a huge increase of the equilibrium concentration of vacancies. At higher hydrogen concentrations $\left(x_{\mathrm{H}}>0.017\right) \mathrm{PdH}$ particles are formed in the matrix. Stresses induced by hydride particles lead to plastic deformation which generates dislocations and vacancies in the sample.

\section{Acknowledgments}

Financial support from the Czech Science Foundation (project P108-13-09436S) and the Ministry of Education of the Czech Republic (project LH12173) is highly acknowledged.

\section{References}

[1] T.B. Flanagan, W.A. Oates, Annu. Rev. Mater. Sci. 21, 269 (1991).

[2] A. Pundt, R. Kirchheim, Annu. Rev. Mater. Res. 36, 555 (2006).

[3] R. Kirchheim, Prog. Mater. Sci. 32, 261 (1988).
[4] T. Mütschele, R. Kirchheim, Scr. Metall. 21, 135 (1987).

[5] F. Besenbacher, J.K. Norskow, M.J. Puska, S. Holloway, Nucl. Instrum. Methods Phys. Res. B 7-8, 55 (1985).

[6] Y. Fukai, N. Ōkuma, Phys. Rev. Lett. 73, 1640 (1994).

[7] R. Kirchheim, Acta Mater. 55, 5129 (2007).

[8] R. Kirchheim, Int. J. Mater. Res. 100, 483 (2009).

[9] P. Hautojärvi, in: Positrons in Solids, Ed. P. Hautojärvi, Springer-Verlag, Berlin 1979, p. 1.

[10] J. Čížek, I. Procházka, F. Bečvář, R. Kužel, M. Cieslar, G. Brauer, W. Anwand, R. Kirchheim, A. Pundt, Phys. Rev. B 69, 224106 (2004).

[11] F. Bečvář, J. Čížek, I. Procházka, J. Janotová, Nucl. Instrum. Methods Phys. Res. A 539, 372 (2005).

[12] I. Procházka, I. Novotný, F. Bečvář, Mater. Sci. Forum 255-257, 772 (1997).

[13] J.M. Campillo Robles, E. Ogando, F. Plazaola, J. Phys., Condens. Matter 19, 176222 (2007).

[14] E. Boroński, R.M. Nieminen, Phys. Rev. B 34, 3820 (1986).

[15] B. Barbiellini, M.J. Puska, T. Korhonen, A. Harju, T. Torsti, R.M. Nieminen, Phys. Rev. B 53, 16201 (1996).

[16] J. Č́žzek, I. Procházka, S. Daniš, G. Brauer, W. Anwand, R. Gemma, E. Nikitin, R. Kirchheim, A. Pundt, R.K. Islamgaliev, Phys. Rev. B 79, 054108 (2009).

[17] C. Borchers, U. Laudahn, A. Pundt, S. Fähler, H.U. Krebs, R. Kirchheim, Philos. Mag. A 80, 543 (2000).

[18] R. West, in: Ref. [9], p. 89.

[19] P. Hautojärvi, C. Corbel, in: Proc. Int. School of Physics "Enrico Fermi", Course CXXV, Eds. A. Dupasquier, A.P. Mills, IOS Press, Varena 1995, p. 491.

[20] H.J. Wollenberger, in: Physical Metallurgy, Vol. 2, Eds. R.W. Cahn, P. Haasen, North-Holland, Amsterdam 1983, p. 1146.

[21] M. Doyama, J.S. Koehler, Acta Metall. 24, 871 (1976). 